\title{
How are countries dealing with their current cardio-vascular disease burden? A snapshot from the WHO Eastern Mediterranean Region (EMR)
}

Director, Noncommunicable Diseases \& Mental Health (NMH) WHO Eastern Mediterranean Regional Office (EMRO) Abdel-Razak El-Sanhouri Street P.O. Box 7608, Nasr City, Cairo 11371, Egypt

*Email: hammericha@who.int http://dx.doi.org/

10.21542/gcsp.2018.1

Received: 20 October 2017 Accepted: 20 December 2017 (C) 2018 The Author(s), licensee Magdi Yacoub Institute. This is an open access article distributed under the terms of the Creative Commons Attribution license CC BY-4.0, which permits unrestricted use, distribution and reproduction in any medium, provided the original work is properly cited.
Asmus Hammerich*

\section{ABSTRACT}

In recent years, a number of global commitments have been made in the area of noncommunicable diseases (NCD). These include the UN NCD Political Declaration in 2011, and the UN Comprehensive Review on NCDs and Outcome Document in 2014. Nine global targets have been agreed in the area of NCDs, and NCDs have been addressed in the Sustainable Development Goals (SDG). Another UN high-level meeting will take place in September 2018 to assess country progress across the globe.

At the regional level, a number of initiates have taken place to deliver on these global commitments. One of the guiding documents is the Regional Framework for Action on Noncommunicable Diseases. This framework was endorsed at the WHO EM Regional Committee in 2012, and includes 17 strategic interventions and 10 monitoring indicators, covering the areas of NCD governance, prevention, surveillance and healthcare.

Progress is being monitored on an annual basis through the development of country progress factsheets and biennial WHO Country Capacity Survey on NCDs. To date however, progress has been insufficient and uneven. Moreover, is has been slowest in the areas of planning and surveillance, and tobacco control.

No uniform approach or model exists for all EMR countries, but a number of countries have advanced their national NCD agenda through original and innovative initiatives.

Perceived challenges include the uneven progress and needs across the WHO EM region, humanitarian emergencies and political instability, vertical approaches, a lack of human and financial resources and other health systems weaknesses.

Opportunities however exist through the global SDG and universal health coverage (UHC) agendas offering an opportunity to revisit essential health services package until 2030.

Overall, there has been political commitment to NCD governance, as evidenced by the EM Regional Committee's endorsement of the regional framework for action. However, despite the clear roadmap, progress has been slow and scattered, differing vastly by country and by topic.

We recommend that countries urgently scale up their efforts in all four areas of the EM Regional Framework of Action to be able to achieve their national and international targets. 


\section{CURRENT WHO EMR COUNTRY STATUS AND PROGRESS OF NONCOMMUNICABLE DISEASES (NCD) AND CARDIO-VASCULAR DISEASES (CVD) PREVENTION AND CONTROL}

In recent years, a number of global commitments have been made in the area of noncommunicable diseases. These include the UN NCD Political Declaration in $2011^{1}$, and the UN Comprehensive Review on NCD and Outcome Document in $2014^{2}$. Nine global targets have been agreed in the area of NCD, and NCD have been addressed in the Sustainable Development Goals (SDG) ${ }^{3}$. The third UN high-level meeting will take place in September 2018, and thus the Eastern Mediterranean Region (EMR) Member States (Figure 2) are proactively working towards achieving the targets.

At the regional level, a number of initiatives have been taken to deliver on these global commitments. One of the guiding documents is the following Regional Framework for Action on Noncommunicable Diseases ${ }^{4}$. This framework was endorsed at the WHO EM Regional Committee in 2012, and includes 17 strategic interventions and 10 monitoring indicators, covering the areas of NCD governance, prevention, surveillance and healthcare (Figure 1).

Progress is being monitored on an annual basis through the development of country progress factsheets on NCDs. The factsheets provide an update for each of the 22 countries, on whether they are fully implementing, partially implementing or not implementing each of the progress 17 sub-indicators. Such information is based on best available evidence, including the data presented in the biennial global WHO Country Capacity Survey on NCDs.

To date, only eight countries in the region are fully achieving six or more of the subindicators; the remaining 14 countries are achieving less than one-third of the subindicators. Progress is slowest in the areas of planning and surveillance, and tobacco control. Therefore the country progress sheets also provide recommendations to each of the 22 countries, on the work elements they should focus on (Source: WHO NCD Global Progress Monitor 20175).

\begin{tabular}{|c|c|c|}
\hline $\begin{array}{l}\text { World Health } \\
\text { Organization }\end{array}$ & \multicolumn{2}{|c|}{$\begin{array}{l}\text { Framework for action to implement the United Nations Political Declaration on } \\
\text { Noncommunicable Diseases, including indicators to assess country progress by } 2018 \\
\text { Updated October 2015, based on resolutions EM/RC59/R.2 \& EM/RC60/R.4 }\end{array}$} \\
\hline Commitments & Strategic interventions & Progress indicators \\
\hline $\begin{array}{l}\text { In the area of } \\
\text { governance }\end{array}$ & 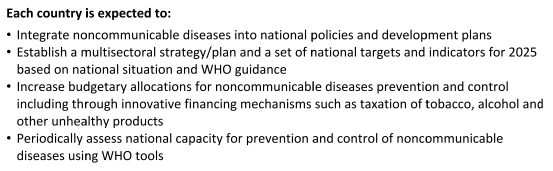 & $\begin{array}{l}\text { Country has: } \\
\text { - An operational multisectoral national strategy/action plan that integrates the major } \\
\text { NCDs and their shared risk factors } \\
\text { - Set time-bound national targets and indicators based on WHO guidance }\end{array}$ \\
\hline Commitments & Strategic interventions & Progress indicators \\
\hline $\begin{array}{l}\text { In the area of } \\
\text { prevention and } \\
\text { reduction of } \\
\text { risk factors }\end{array}$ & $\begin{array}{l}\text { Each country is expected to: } \\
\text { - Accelerate implementation of the WHO Framework Convention on Tobacco Control (WHO } \\
\text { FCTC) and ratify Protocol to Eliminate Illicit Trade in Tobacco Products } \\
\text { - Ensure healthy nutrition in early life and childhood including breastfeeding promotion and } \\
\text { regulating marketing of foods and non-alcoholic beverages to children } \\
\text { - Reduce average population salt intake in line with WHO recommendations } \\
\text { - Virtually leliminate transfat intake and reduce intake of saturated fatty acids } \\
\text { - Promote physical activity through a life-course approach } \\
\text { - Implement the best buys to reduce the harmful use of alcohol }\end{array}$ & $\begin{array}{l}\text { Country is implementing: } \\
\text { - Four demand-reduction measures of the WHO FCTC at the highest level of achievement } \\
\text { - Four measures to reduce unhealthy diet } \\
\text { - At least one recent national public awareness programme on diet and/or physical } \\
\text { activity } \\
\text { - As appopriate, according to national circumstances, three measures to reduce the } \\
\text { harmful use of alcohol, in line with the WHO global strategy to reduce the harmful use } \\
\text { of alcohol }\end{array}$ \\
\hline Commitments & Strategic interventions & Progress indicators \\
\hline $\begin{array}{l}\text { In the area of } \\
\text { surveillance, } \\
\text { monitoring and } \\
\text { evaluation }\end{array}$ & $\begin{array}{l}\text { Each country is expected to: } \\
\text { - Implement/strengthen the WHO surveillance framework that monitors mortality and } \\
\text { morbidity, risk factors and detetrminants, and health system capacity and response } \\
\text { - Integerate the three components of the surveillance framework into the national health } \\
\text { information system } \\
\text { - Strengthen human resources and institutional capacity for surveillance, monitoring and } \\
\text { evaluation }\end{array}$ & $\begin{array}{l}\text { Country has: } \\
\text { - A functioning system for generating reliable cause-specific mortality data on a routine } \\
\text { basis } \\
\text { - A STEPS survey or a comprehensive health examination survey every } 5 \text { years }\end{array}$ \\
\hline Commitments & Strategic interventions & Progress indicators \\
\hline $\begin{array}{l}\text { In the area of } \\
\text { health care }\end{array}$ & $\begin{array}{l}\text { Each country is expected to: } \\
\text { - Implement the best buys in health care } \\
\text { - Improve access to early detection and management of major noncommunicable diseases } \\
\text { and risk factors by including them in the essential primary heath hare package } \\
\text { - Improve access to safe, affordable and quality essential medicines and technologies for } \\
\text { major noncommunicale diseases } \\
\text { - Improve access to essential palliative care services }\end{array}$ & $\begin{array}{l}\text { Country has: } \\
\text { - Evidence-based national guidelines/protocols/standards for management of major } \\
\text { noncommunicable diseases through a primary care approach, recognized/approved by } \\
\text { the government or competent authority } \\
\text { - Provision of drug therapy, including glycaemic control, and counselling for eligible } \\
\text { persons at high hirk to prevent heart attacks and strokes, with an emphasis on the } \\
\text { primary care level }\end{array}$ \\
\hline
\end{tabular}

Figure 1. Regional Framework for Action on Noncommunicable Diseases. 


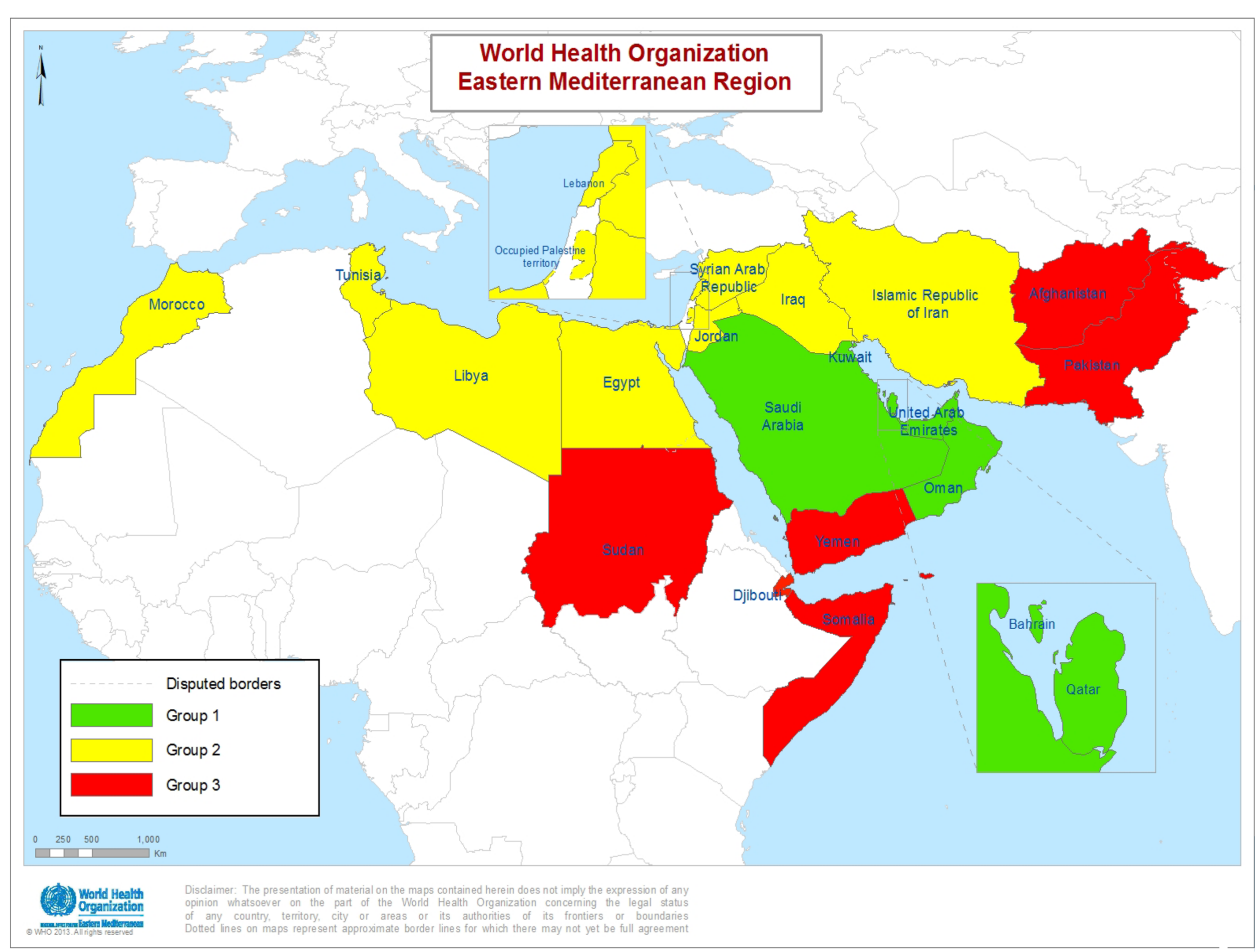

Figure 2. Member States of the WHO Eastern Mediterranean Region, color-coded by population health outcomes, health system performance and level of health expenditure in 2017. (Group 1 comprises countries where socioeconomic and health development has progressed considerably over the past decades. Group 2 comprises largely middle-income countries which have developed extensive public health infrastructure but face resource constraints. Group 3 comprises countries which face constraints in improving population health outcomes as a result of lack of resources, political instability and other complex development challenges).

For monitoring national NCD response, capacity for NCD or CVD management has been assessed in terms of the existence of operational multi-sectoral national strategies or action plans integrating the major NCDs and their shared risk factors, evidence-based national guidelines, protocols or other standards for the management and referral of major NCD (recognized or approved by government or competent authorities), the availability of nine essential NCD medicines at the primary care level, and the provision of drug therapy, including glycemic control and counselling for eligible persons at high risk to prevent heart attacks and stroke 5 .

\section{EXPERIENCES FOR CVD PREVENTION AND CONTROL IN THE WHO EMR}

No uniform approach or model exists for all EMR countries. Longstanding vertical programs on hypertension and diabetes mellitus with country tools are in place, but limited integration and focus on total CVD risk, monitoring of health system performance, uptake and sustainable use of WHO package of essential NCD interventions for primary health care (PEN) persist. Also, a 'health system lens' to identify and address key bottlenecks is missing in many countries. However there appears to be a renewed interest in the launch of Global HEARTS initiative. Some encouraging EMR country experiences include Palestine, Oman, Bahrain (WHO PEN protocol implementation) and Iran ('IraPEN' pilot implementation through community health workers (Community health workers (CHW) or 'bevharzes') and the Iran CVD-SUPPORT Trial).

The example of IraPEN has a number of outstanding features such as a well-designed package of interventions covering the four main NCD and their related risk factors 
adapted from WHO PEN; good clinical pathways for early detection CVD, three priority cancers and asthma delivered through a multidisciplinary team (CHW, midwifes, family practitioners); coordinated care based on defined tasks with support of other disciplines relevant for NCD care (mental health, nutrition) and strong focus on self-care; CHW's and midwives' expanded scope of work with demonstrated pilot feasibility of IRA PEN model (good knowledge, skills to assess, advice and manage cardio-vascular risk and other NCD); good availability, affordability of essential medicines and technologies; and clear organizational structures from primary care centers to central level for oversight and support of IraPEN, with health Information management tools being available for programme monitoring at different levels ${ }^{6}$.

\section{CHALLENGES \& OPPORTUNITIES IN THE EMR}

Perceived challenges include the uneven progress and needs across the WHO EM region; humanitarian emergencies and political instability; vertical approaches and programs; a lack of human and financial resources (both in ministries of health and WHO); and other health systems weaknesses such as poor capacity for guideline adaptation and development of tools; availability or affordability of NCD medicines; training and supportive supervision; NCD related data analysis; insufficient and unsustainable country support for NCD integration; and WHO PEN advocacy and communication.

Opportunities however exist through the global SDG and universal health coverage (UHC) agendas offering an opportunity to revisit essential health services package until 2030; a willingness to prioritize cost-effective interventions (e.g., WHO NCD Global Action Plan, Appendix 3, Update 2017) until 2020; the systematic NCD and CVD monitoring within the Global NCD Monitoring Framework until 2025; a better alignment with health sector reform and strengthening efforts and other integrated service delivery initiatives as well as the 'One UN' and 'One WHO Integrated Country Support' momentum e.g., UN NCD interagency taskforce and investment case missions?.

\section{CONCLUSIONS AND RECOMMENDATIONS}

Overall, there has been strong political commitment to NCD governance, as evidenced by the EM Regional Committee's endorsement of the regional framework for action. However, despite the clear roadmap, progress has been slow and scattered, differing vastly by country and by topic. The factors inhibiting progress include the complex emergencies affecting many countries in the region, in addition to economic constraints. Nevertheless, the WHO tools and frameworks have been well received across the region and are vital documents to support countries in the development, implementation and monitoring of their NCD policies, strategies and plans. The WHO EM regional office continues to provide technical support to its 22 countries, strongly supporting them in achieving the targets they have pledged to attain.

Specifically for CVD governance, national policies, strategies and plans, targets and indicators, stewardship, advocacy and information sharing should be further promoted. For CVD prevention and reduction of risk factors, the evidence-based NCD 'best-buys' for tackling tobacco use (i.e., WHO FCTC and MPOWER package) and alcohol abuse (where appropriate), unhealthy nutrition (excessive salt, trans-fatty acids, sugar intake) and physical inactivity have to be tackled more vigorously. In the area of CVD management and health care the full implementation of the recommendations of Global HEARTS initiative with a focus on treatment protocols at primary health care level should be propagated. Finally, in the field of CVD surveillance, monitoring and evaluation, countries 
are encouraged to regularly conduct NCD country capacity, STEP and other risk factor surveys, to generate more reliable cause-specific mortality data, and to agree on key performance indicators at facility level (making use of the Global HEARTS monitoring and evaluation module).

\section{REFERENCES}

[1] United Nations (UN) General Assembly. Political Declaration on NCD. New York City, USA: UN Headquarters publications; 2011.

[2] United Nations (UN) General Assembly. Comprehensive Review and Outcome Document on NCD. New York City, USA: UN Headquarters publications; 2014.

[3] United Nations (UN). Sustainable Development Goals (SDG): NCD targets and indicators. New York City, USA: UN Headquarters publications; 2016.

[4] World Health Organization (WHO). Eastern Mediterranean Regional (EMR) Framework for Action on NCD. Cairo, Egypt: WHO EMRO publications; 2012.

[5] World Health Organization (WHO). Global Progress Monitor on NCD. Geneva, Switzerland: WHO Headquarters publications; 2017.

[6] The IraPEN experience in the Islamic Republic of Iran. Tehran, Islamic Republic of Iran: Ministry of Health Iran; 2017.

[7] United Nations (UN). NCD interagency taskforce and investment case missions. Geneva, Switzerland: WHO Headquarters; 2017. 